\title{
Androgen Receptor, EGFR, and BRCA1 as Biomarkers in Triple-Negative Breast Cancer: A Meta-Analysis
}

\author{
Li Zhang, ${ }^{1}$ Cheng Fang, ${ }^{1}$ Xianqun Xu, ${ }^{1}$ Anling Li, ${ }^{1}$ Qing Cai, ${ }^{2}$ and Xinghua Long ${ }^{1}$ \\ ${ }^{1}$ Zhongnan Hospital of Wuhan University, Wuhan 430071, China \\ ${ }^{2}$ Hubei University of Chinese Medicine, Wuhan 430061, China \\ Correspondence should be addressed to Xinghua Long; xlong888@yahoo.com
}

Received 18 July 2014; Revised 5 November 2014; Accepted 7 November 2014

Academic Editor: Gagan Deep

Copyright (c) $2015 \mathrm{Li}$ Zhang et al. This is an open access article distributed under the Creative Commons Attribution License, which permits unrestricted use, distribution, and reproduction in any medium, provided the original work is properly cited.

\begin{abstract}
Objective. More and more evidences demonstrate that androgen receptor (AR), epidermal growth factor receptor (EGFR), and breast cancer susceptibility gene 1 (BRCA1) have unique clinical implications for targeted therapy or prognosis in triple-negative breast cancer (TNBC). Therefore, we conducted a meta-analysis to summarize the possible associations. Methods. We retrieved published articles about AR, EGFR, and BRCA1 in TNBC from PubMed and EMBASE. The analysis was performed with Rev-Man 5.2 software. Results. A total of 38 articles were eligible for the meta-analysis. Our study showed that the expression level of EGFR $(\mathrm{OR}=6.88, P<0.00001)$ and the prevalence of BRCA1 mutation $(\mathrm{RR}=5.26, P<0.00001)$ were higher in TNBC than non-TNBC. In contrast, the expression level of AR was lower in TNBC than non-TNBC (OR $=0.07, P<0.00001)$. In the subgroup related to EGFR expression, the level of EGFR expression was significantly increased in Asians $(\mathrm{OR}=9.60)$ compared with Caucasians $(\mathrm{OR}=5.53)$ for TNBC patients. Additionally, the prevalence of BRCA1 mutation in Asians $(\mathrm{RR}=5.43, P<0.00001)$ was higher than that in Caucasians $(\mathrm{RR}=5.16, P<0.00001)$. Conclusions. The distinct expression of AR and EGFR and the prevalence of BRCA1 mutation indicated that AR, EGFR, and BRCA1 might be unique biomarkers for targeted therapy and prognosis in TNBC.
\end{abstract}

\section{Introduction}

As one of the most common malignant tumors in female patients worldwide, breast cancer is recognized as a heterogeneous cancer, which shows substantial diversities related to biological behavior, therapeutic response, and clinical outcome [1]. Gene expression analysis can make breast cancers fall into at least 5 subtypes that are luminal A, luminal B, normal-like, basal-like, and human epidermal growth factor receptor 2 overexpression by using DNA microarrays [1-3]. Besides having different molecular pathology and clinical manifestation, different subtypes have different response to treatments. Triple-negative breast cancer (TNBC) characterized by the absence of estrogen receptor alpha (ER) and progesterone receptor (PR) expression and HER2 overexpression is mostly basal-like subgroup of breast cancers $[1,3]$ and accounts for $10 \%-20 \%$ of all breast cancers [4]. Conventional hormonal or anti-HER2 targeted therapies have no favorable value for TNBC which lacks known common therapeutic targets. Moreover, TNBC patients have recurrence rate of
$6.7 \%-10.5 \%$ which is higher compared with an overall rate of $2.1 \%-6.4 \%$ among all breast cancer patients and have shorter times to recurrence ranging from 19 to 40 months versus 35 to 67 months in non-TNBC patients [5]. Therefore, aggressive clinical behavior and poor prognosis make it urgent to search for appropriate biomarkers for effective treatment options and judgment of prognosis.

As a subtype of breast cancer, TNBC is also a highly diverse group of cancers with unique molecular subtypes, which have distinct clinicopathologic characteristics and react dissimilarly to targeted agents and chemotherapy. By analyzing gene expression profiles, Lehmann et al. [4] identified 6 TNBC molecular subtypes with distinct characteristics which included two basal-like (BL1 and BL2), an immunomodulatory (IM), a mesenchymal-like (M), a mesenchymalstem-like (MSL), and a luminal androgen receptor (LAR) subtype. The BL1 and BL2 were enriched in DNA damage response (ATR/BRCA) pathways and cell cycle genes (e.g., EGFR), and the LAR subtype was featured by androgen receptor (AR) signaling. Park et al. [6] demonstrated that 
BRCA1 was a coactivator of AR and might directly modulate AR signaling. Furthermore, a recent study showed that AR expression correlated with activated membrane receptor kinase EGFR in TNBC patients, and concomitant administration of anti-androgen bicalutamide with EGFR inhibitor decreased the amount of AR, along with an antiproliferative effect [7]. Moreover, BRCA1-related breast cancer is associated with a basal-like phenotype in which EGFR is a basal marker [2] and approximately one in five BRCA1 mutated breast cancers which were negative for ER and PR expressed AR [8]. Therefore, there are certain interaction relations between AR, EGFR, and BRCA1 in the initiation and progression of TNBC, which have not been fully investigated. However, it may be worthwhile carrying out more studies regarding the unique functions of these biomarkers in TNBC.

Compared with ER or PR in breast cancers, few researches are conducted for the roles of androgen and androgen receptor (AR) in breast cancers. AR, a member of the steroid hormone receptor family, is reported to be expressed in more than $70 \%$ of breast cancer $[9,10]$ and detected only in $25-35 \%$ of TNBCs $[10,11]$. Low expression of AR is associated with distant metastasis in the AR-positive TNBCs [12]. Previous studies about AR expression indicated that AR-negative TNBC showed significantly poorer outcomes with regard to the disease-free survival and overall survival than the ARpositive TNBC $[1,13]$, which suggested that AR expression could be a valuable prognostic marker in TNBC.

In general, not all TNBCs have a basal-like phenotype and not all basal-like breast cancers are TNBCs [2], but TNBC takes the place of the basal-like breast cancer in the application of clinical diagnosis and treatment as a result of realistic feasibility that immunohistochemical method is more feasible for large-scale clinical application or retrospective studies than gene expression signature. Therefore, EGFR, as a basal marker and a transmembrane receptor on the cell surface, can have distinctive expression level and functions in TNBCs. More and more emerging data demonstrated that the expression level of EGFR was increased especially in TNBC $[1,10$, 14]. In addition, its expression has been displayed to be concerned in neoplasms proliferation, invasion, and angiogenesis [15]. Thus, EGFR may play a distinct role in targeted therapy for specific inhibitors and prognosis in TNBC.

The cancer suppressor gene BRCA1 is involved in the process of DNA damage repair, recombination, cell cycle, and transcription [16]. Intriguingly, BRCA1-mutated tumors are correlated with the basal-like phenotype $[2,17]$. Moreover, it is believed that BRCA1 mutation accounts for the progress of hereditary breast cancers and is in connection with unique clinicopathological characteristics compared with sporadic breast cancers [18]. Emerging data demonstrate that BRCA1 mutation is more likely to be identified in TNBC compared with non-TNBC, with BRCA1 mutation rate of $50 \%-87 \%$ in TNBC patients [3, 18-21]. Therefore, the hypothesis should be taken into account that there is an association between BRCA1 mutation carriers, basal-like phenotype, and TNBC, revealing a new angle for the research into the treatment and prognosis in TNBCs.

It is the interest of studies for AR expression, EGFR expression, and BRCA1 mutation in TNBC patients, which offer help for treatment options or prognosis for TNBCs. Although the three biomarkers have been intensively studied, most of the research studied them, respectively, and the research results were not fully consistent. Thus, we performed a meta-analysis to systematically evaluate AR expression, EGFR expression, and the risk of BRCA1 mutation in TNBC.

\section{Material and Methods}

2.1. Publication Search. We collected literature by searching PubMed and EMBASE, with a combination of the following keywords: "AR and triple-negative breast cancer," "EGFR and triple-negative breast cancer," and "BRCA1 mutation and triple-negative breast cancer," respectively, up to May 2014. We evaluated potentially relevant literature by scanning their titles and abstracts, or even full texts in the condition of having no idea about eligibility for publications. In addition, we paid an attention to the references of the qualified articles to see if there were more eligible ones.

2.2. Inclusion Criteria. Candidate studies included in the meta-analysis had to satisfy all the following criteria: related to AR or EGFR or BRCA1 in TNBC; use a case-control design; sufficient published data for estimating an odds ratio (OR) or risk ratio (RR) with $95 \%$ confidence interval (CI); being limited to human subjects.

2.3. Excluding Criteria. The following criteria were used to exclude published studies: reviews and letters; lack of key information (the total number in TNBC group and nonTNBC group; the event number about the AR expression or EGFR expression or BRCA1 mutation in TNBC and nonTNBC) for calculating OR for the expression of AR and EGFR or RR for the risk of BRCA1 mutation and 95\% CI; nonEnglish articles.

2.4. Data Extraction. For each of the eligible articles, the following data was extracted independently by two researchers (Zhang and Fang): first author's surname, year of publication, study origin, study objects, measuring method, positive judgement standards, and so on (Tables 1, 2, and 3).

2.5. Statistical Analysis. Crude ORs with their 95\% CI were used to assess the strength of association between the expression of AR and TNBC and EGFR expression and TNBC, respectively. The same was $\mathrm{RR}$ and $95 \% \mathrm{CI}$ for the risk of BRCA1 mutation in TNBC. The significance of the pooled OR or RR was determined by the $Z$-test, and $P<0.05$ was considered as statistically significant. Pooled OR or RR was carried out with Review Manager 5.2 software recommended by Cochrane Collaboration. In this meta-analysis, we define the exposed group as triple-negative breast cancer (TNBC) and nonexposed group as non-triple-negative breast cancer (nonTNBC). There are three parts in our analysis: one is for AR expression and TNBC; one is for EGFR expression and TNBC; and the last is for having the risk of BRCA1 mutation in TNBC.

Heterogeneity in meta-analysis is concerned with the variation in research outcomes among different literature. 
TABLE 1: Main characteristics of all studies included in the meta-analysis for AR.

\begin{tabular}{|c|c|c|c|c|c|c|}
\hline References & Year & Origin & Case-controls & Antibody source & Dilution & Cut-off value \\
\hline Rakha et al. [1] & 2007 & UK & $277 / 1370$ & BioGenex & $1: 30$ & $>0 \%$ \\
\hline Pristauz et al. [8] & 2010 & Austria & $44 / 91$ & Dako & $1: 100$ & Not available \\
\hline Gasparini et al. [10] & 2014 & USA & $371 / 256$ & Dako & $1: 100$ & $>5 \%$ \\
\hline Chae et al. [22] & 2011 & Korea & $12 / 157$ & Dako & Not available & $>10$ \\
\hline Hu et al. [23] & 2011 & USA & $211 / 1256$ & Dako & $1: 200$ & $>1 \%$ \\
\hline Koo and Jung [24] & 2011 & Korea & $8 / 109$ & Lab Vision Corp & $1: 100$ & Not available \\
\hline Luo et al. [25] & 2010 & China & $137 / 132$ & Zymed & $1: 100$ & $>1 \%$ \\
\hline Loibl et al. [26] & 2011 & Germany & $111 / 562$ & Dako & $1: 150$ & $>1 \%$ \\
\hline Micello et al. [27] & 2010 & Italy & $138 / 88$ & Novocastra & $1: 20$ & $>10 \%$ \\
\hline Niemeier et al. [28] & 2010 & USA & $30 / 159$ & Dako & $1: 50$ & $>10 \%$ \\
\hline Ogawa et al. [29] & 2008 & Japan & $42 / 185$ & Dako & $1: 100$ & $>10 \%$ \\
\hline Park et al. [30] & 2009 & Korea & $63 / 350$ & Dako & Not available & $>10 \%$ \\
\hline Park et al. [31] & 2011 & Korea & $156 / 775$ & Thermo Scientific & Not available & $>10 \%$ \\
\hline Peters et al. [32] & 2012 & Australia & $18 / 36$ & Not available & Not available & Not available \\
\hline
\end{tabular}

TABLE 2: Main characteristics of all studies included in the meta-analysis for EGFR.

\begin{tabular}{|c|c|c|c|c|c|c|}
\hline References & Year & Origin & Case-controls & Antibody source & Dilution & Cut-off value \\
\hline Rakha et al. [1] & 2007 & UK & $249 / 1293$ & Novocastra & $1: 10$ & $>10 \%$ \\
\hline Gasparini et al. [10] & 2014 & USA & $381 / 262$ & Dako & $1: 100$ & $>10 \%$ \\
\hline Tang et al. [14] & 2012 & China & $40 / 158$ & Dako & Not available & $>0 \%$ \\
\hline Koo and Jung [24] & 2011 & Korea & $8 / 109$ & Novocastra & $1: 50$ & $>10 \%$ \\
\hline Nogi et al. [33] & 2009 & Japan & $26 / 85$ & Novocastra & Not available & $>0 \%$ \\
\hline Nozoe et al. [34] & 2011 & Japan & $7 / 30$ & Dako & $1: 10$ & $>10 \%$ \\
\hline Pillai et al. [35] & 2012 & Malaysia & $18 / 18$ & Dako & $1: 50$ & $>1 \%$ \\
\hline Rydén et al. [36] & 2010 & Sweden & $87 / 299$ & Dako & Prediluted & $>1 \%$ \\
\hline Tan et al. [37] & 2008 & UK & $31 / 209$ & Zymed & $1: 50$ & Not available \\
\hline Tawfik et al. [38] & 2010 & USA & $151 / 379$ & Zymed & $1: 20$ & Not available \\
\hline
\end{tabular}

TABLE 3: Main characteristics of all studies included in the meta-analysis for BRCA1.

\begin{tabular}{|c|c|c|c|c|}
\hline References & Year & Origin & Case-controls & Method of BRCA1 testing \\
\hline Haffty et al. [3] & 2006 & USA & $34 / 65$ & Not mentioned \\
\hline Pristauz et al. [8] & 2010 & Austria & $44 / 91$ & MLPA \\
\hline Atchley et al. [18] & 2008 & USA & $93 / 384$ & Not mentioned \\
\hline Musolino et al. [19] & 2007 & Italy & $20 / 26$ & DHPLC \\
\hline Li et al. [20] & 2008 & China & $17 / 61$ & SSCP, DHPLC \\
\hline Byrski et al. [21] & 2008 & Poland & $28 / 37$ & Multiplex allele-specific PCR \\
\hline Bayraktar et al. [39] & 2013 & USA & $44 / 108$ & Not mentioned \\
\hline Chen et al. [40] & 2009 & China & $25 / 107$ & DHPLC, germline DNA \\
\hline Comen et al. [41] & 2011 & USA & $64 / 387$ & pyrosequencing \\
\hline Kwong et al. [42] & 2009 & China & $59 / 146$ & MLPA \\
\hline Lee et al. [43] & 2011 & Norway & $156 / 1011$ & PCR \\
\hline Noh et al. [44] & 2013 & Korea & $52 / 178$ & PCR, Sequencher software \\
\hline Ou et al. [45] & 2013 & China & $24 / 55$ & PCR-DHPLC \\
\hline Phuah et al. [46] & 2012 & Japan & $110 / 321$ & MLPA \\
\hline $\mathrm{Xu}$ et al. [47] & 2012 & China & $76 / 276$ & HRM-PCR \\
\hline Yip et al. [48] & 2009 & Malaysia & $23 / 58$ & MLPA \\
\hline Zhang et al. [49] & 2012 & China & $96 / 271$ & PCR, BigDye \\
\hline Rakha et al. [50] & 2009 & UK & $27 / 216$ & Not mentioned \\
\hline
\end{tabular}




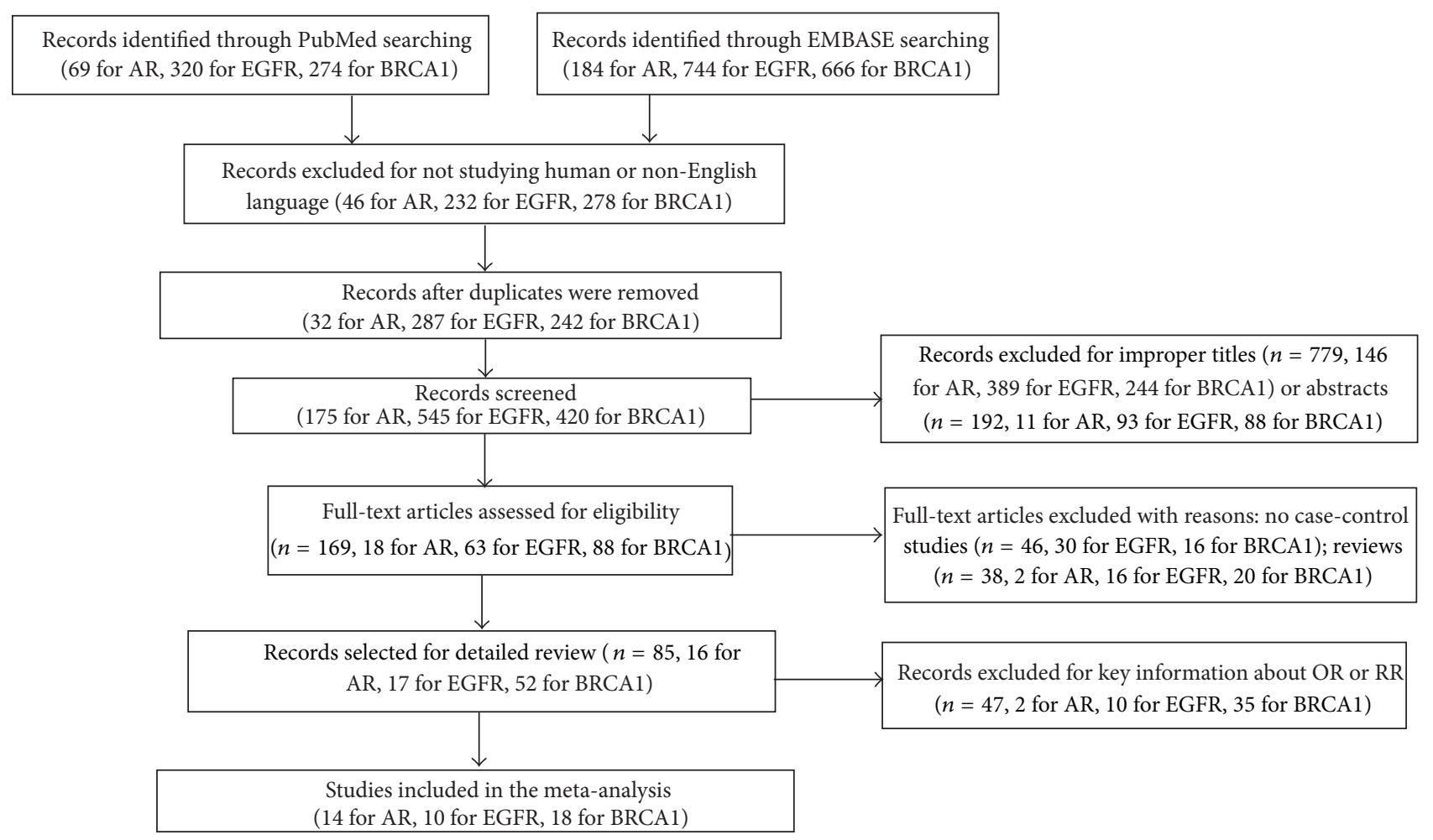

Figure 1: Flow diagram of the study.

The Q-statistic was applied to investigate heterogeneity among studies with Review Manager 5.2 software. $P$ value greater than 0.1 for $Q$-test indicated the absence of heterogeneity, and the fixed-effect model (Mantel-Haenszel method) was used to calculate pooled ORs. Otherwise, heterogeneity was present and the random-effect model (DerSimonian-Laird method) was more appropriate. In addition, the $I^{2}$-test put forward by Higgins and Thompson was employed to accurately measure the degree of heterogeneity [51]. The value of $I^{2}$ ranges from $0 \%$ to $100 \%$, and $I^{2}=0 \sim 25 \%$ implied no heterogeneity, $I^{2}=25 \sim 50 \%$ moderate heterogeneity, $I^{2}=50 \sim 75 \%$ large heterogeneity, and $I^{2}=75 \sim 100 \%$ extreme heterogeneity [52]. Publication bias of literature was evaluated by funnel plot, and symmetry of the funnel plot was considered to lack statistically significant publication bias.

\section{Results}

3.1. Characteristics of Eligible Literature. We retrieved the published literature about AR, EGFR, and BRCA1 in TNBC from PubMed and EMBASE. After being manually filtered in accordance with the inclusion and exclusion criteria, a total of 14 studies [1, 8, 10, 22-32] involving 1,618 cases and 5,526 controls were analyzed for AR, 10 studies $[1,10,14,24,33-$ 38] involving 998 cases and 2,842 controls for EGFR, and 18 studies [3, 8, 18-21, 39-50] involving 992 cases and 3,798 controls for BRCA1. The flow diagram of the study was shown in Figure 1. There were 3 articles related to AR and EGFR [1, $10,24]$ and 1 article concerned with AR and BRCA1 [8]. Therefore, there were in total 38 articles for 42 studies. All studies detected the expression level of AR and EGFR by using immunohistochemical method. However, besides the antibody source and the dilution ratio, it was notable that there was no universally accepted standard about the cut-off value of the low expression for AR or overexpression for EGFR. In addition, different methods were applied for the BRCA1 testing, such as PCR-SSCP, MLPA, and DHPLC. The main characters of eligible studies, such as the first author's name, publication date, sources of research, sample size for casecontrol, antibody source, dilution ratio, cut-off value, and method of BRCA1 testing, were summarized in Tables 1-3, respectively.

3.2. The Results of Meta-Analysis. The main results of this meta-analysis were showed in Figures 2, 3, 4, 5, and 6.

For AR, significantly low expression was observed in TNBC (pooled OR $=0.07,95 \% \mathrm{CI}=0.05-0.11$ ), and there was statistical significance $(P<0.00001)$, which we could see in Figure 2. Random-effect model was chosen for AR expression on account of extreme heterogeneity $\left(P<0.00001, I^{2}=\right.$ $79 \%)$. In the stratified analysis by ethnic groups, the result showed that AR expression was slightly increased in Asians $(\mathrm{OR}=0.11)$ compared with Caucasians $(\mathrm{OR}=0.06)$ and the details were shown in Table 4.

The analysis of EGFR expression in TNBC was performed and it showed extreme heterogeneity $\left(I^{2}=85 \%, P<\right.$ 0.00001 ) among studies, so that a random-effect model was applied to calculate a pooled OR $(6.88,95 \% \mathrm{CI}=3.84-12.35)$. As shown in Figure 3, the expression level of EGFR was statistically significantly higher in TNBC than non-TNBC 


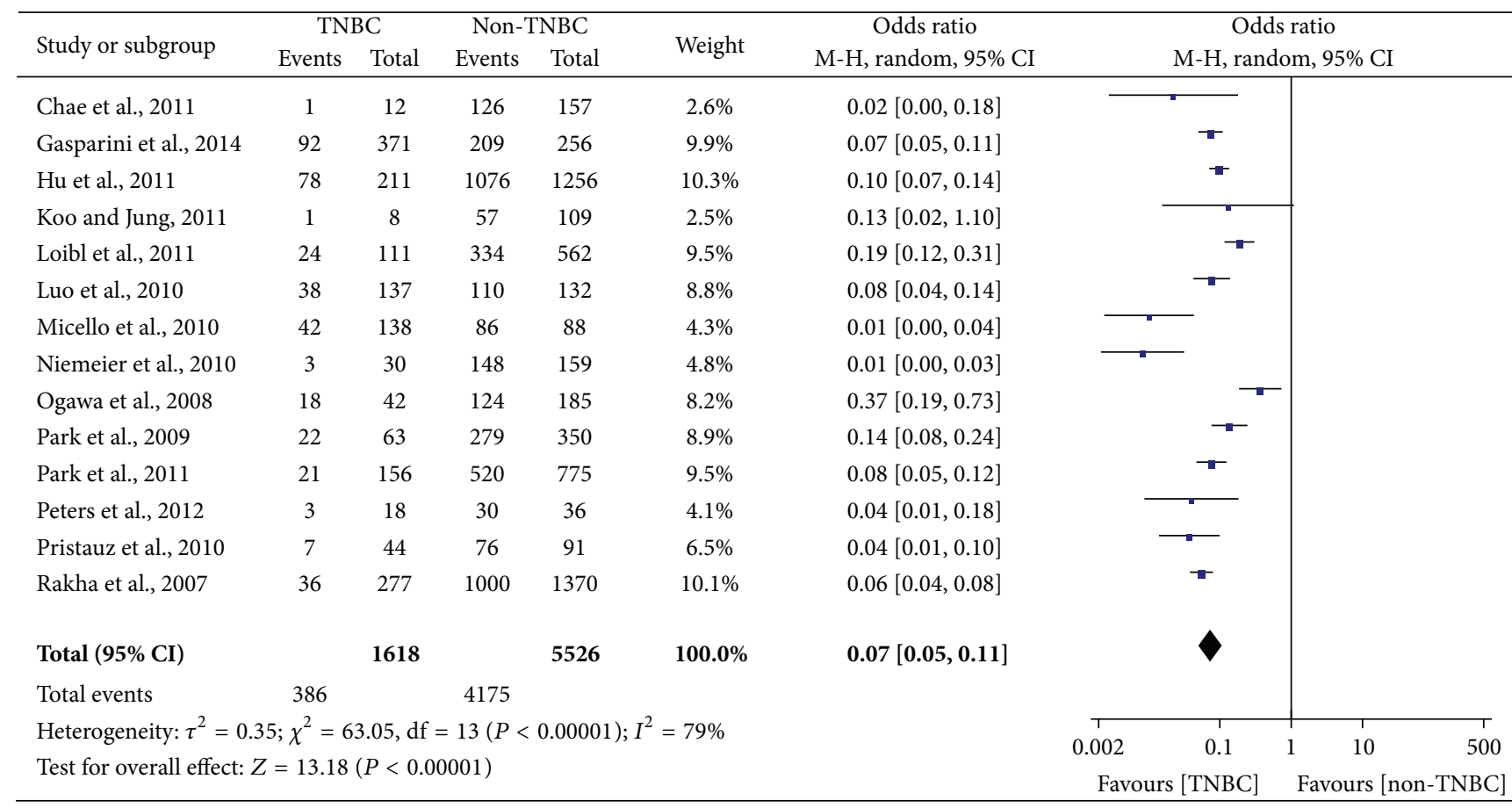

FIGURE 2: Forest plot of studies evaluating OR of AR expression in TNBC compared with non-TNBC. The events of TNBC and the events of non-TNBC refer to the number of TNBC patients with positive expression of AR and the number of non-TNBC patients with positive expression of AR, respectively. The squares and horizontal lines correspond to the specific OR and 95\% CI for every study. The area of the squares reflects the study specific weight. The diamond stands for the pooled OR and 95\% CI.

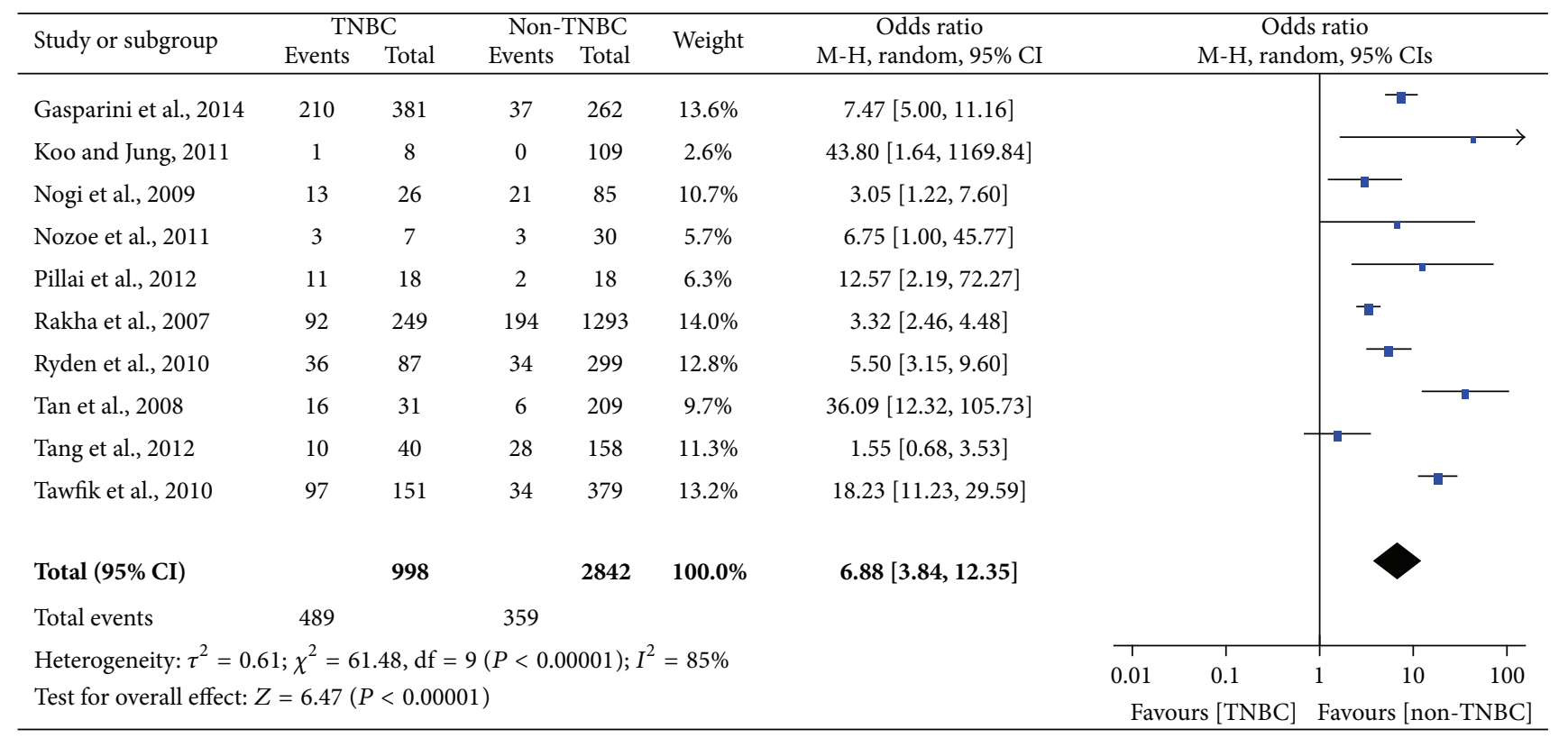

FIGURE 3: Forest plot of studies evaluating OR of EGFR expression in TNBC compared with non-TNBC. The events of TNBC and the events of non-TNBC refer to the number of TNBC patients with positive expression of EGFR and the number of non-TNBC patients with positive expression of EGFR, respectively. The squares and horizontal lines correspond to the specific OR and 95\% CI for every study. The area of the squares reflects the study specific weight. The diamond stands for the pooled OR and 95\% CI. 


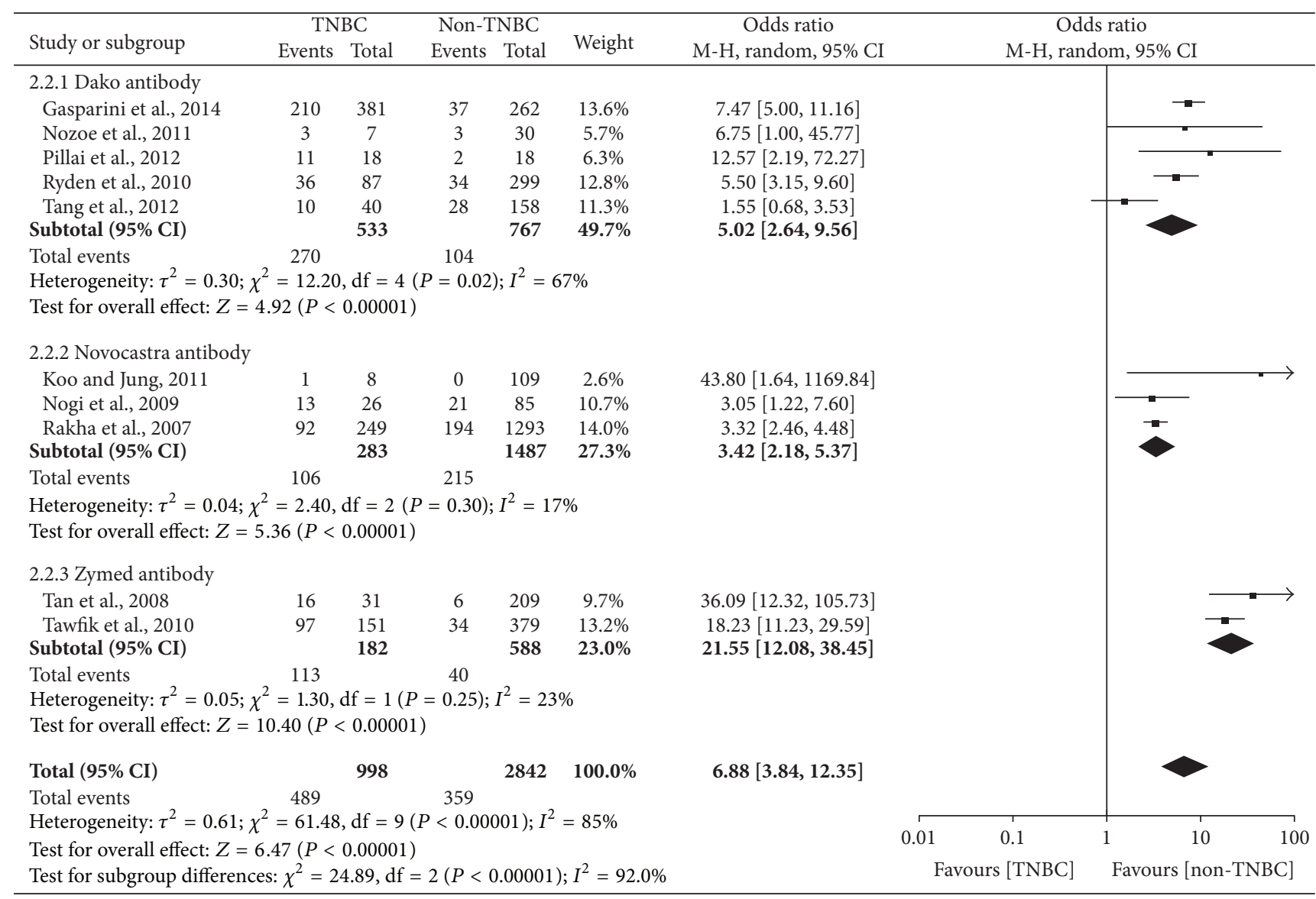

FIGURE 4: Forest plot of subgroup related to antibody source evaluating EGFR expression in TNBC compared with non-TNBC.

TABLE 4: Subgroup analyses based on ethnic for AR expression, EGFR expression, and BRCA1 mutation in TNBC compared with non-TNBC.

\begin{tabular}{|c|c|c|c|c|c|c|c|c|c|c|c|c|}
\hline \multirow{2}{*}{ Variables } & \multicolumn{4}{|c|}{$\mathrm{AR}$} & \multicolumn{4}{|c|}{ EGFR } & \multicolumn{4}{|c|}{ BRCA1 } \\
\hline & $N$ & OR $(95 \% \mathrm{CI})$ & $P_{H}$ & $I^{2}$ & $N$ & OR $(95 \% \mathrm{CI})$ & $P_{H}$ & $I^{2}$ & $N$ & RR $(95 \% \mathrm{CI})$ & $P_{H}$ & $I^{2}$ \\
\hline Total & 14 & $0.07(0.05-0.11)$ & $<0.001$ & $79 \%$ & 10 & $6.88(3.84-12.35)$ & $<0.001$ & $85 \%$ & 18 & $5.26(4.42-6.26)$ & 0.14 & $27 \%$ \\
\hline \multicolumn{13}{|l|}{ Ethnic } \\
\hline Asian & 6 & $0.11(0.06-0.21)$ & 0.002 & $73 \%$ & 5 & $9.60(3.50-26.30)$ & 0.01 & $68 \%$ & 9 & $5.43(4.06-7.25)$ & 0.71 & $0 \%$ \\
\hline Caucasian & 8 & $0.06(0.03-0.09)$ & $<0.001$ & $82 \%$ & 5 & $5.53(2.84-10.77)$ & $<0.001$ & $87 \%$ & 9 & $5.16(4.16-6.40)$ & 0.02 & $55 \%$ \\
\hline
\end{tabular}

$N$ : numbers of data sets; $P_{H}: P$ value of $Q$-test for heterogeneity test; $P_{H}<0.1$ indicates that there is heterogeneity and random-effect model is used to calculate pooled OR or RR and $95 \%$ CI. Otherwise, fixed-effect model is used.

$(P<0.00001)$. Because of the extreme heterogeneity, we did a subgroup analysis according to the antibody source and the result was shown in Figure 4 . The heterogeneity was markedly reduced in the subgroup and it indicated that the antibody source was one of the factors for the extreme heterogeneity. Moreover, we also did stratified analysis related to ethnicity, and it was worth noting that EGFR expression was significantly increased in Asians $(\mathrm{OR}=9.60)$ compared with Caucasians $(\mathrm{OR}=5.53)$ for TNBC patients (Table 4$)$.

In Figure 5, the outcome of heterogeneity for the risk of BRCA1 mutation in TNBC was that $I^{2}=27 \%$ and $P_{\text {heterogeneity }}=0.14$, so a fix effect model was used to calculate the pooled RR $(5.26,95 \% \mathrm{CI}=4.42-6.26)$. It indicated that women with TNBC were approximately five times more likely to have BRCA1 mutation compared with non-TNBC and it was statistically significant $(P<0.00001)$. In the subgroup with regard to ethnicity, the pooled $\mathrm{RR}(5.43,95 \% \mathrm{CI}=$ 4.06-7.25) for the Asians was higher than the pooled RR (5.16, 95\% CI $=4.16-6.40$ ) for the Caucasians, which indicated that the prevalence of BRCA1 mutation was higher in the Asians compared with the Caucasians (Figure 6).

Finally, publication bias of the eligible studies was evaluated by Funnel plots, respectively. As shown in Figure 7, the funnel plots about AR and EGFR were almost symmetric, which meant that there was absent indication for significant publication bias. However, there was slight asymmetry in the funnel plot about BRCA1, which indicated that there was mild publication bias between the eligible articles about BRCA1. 


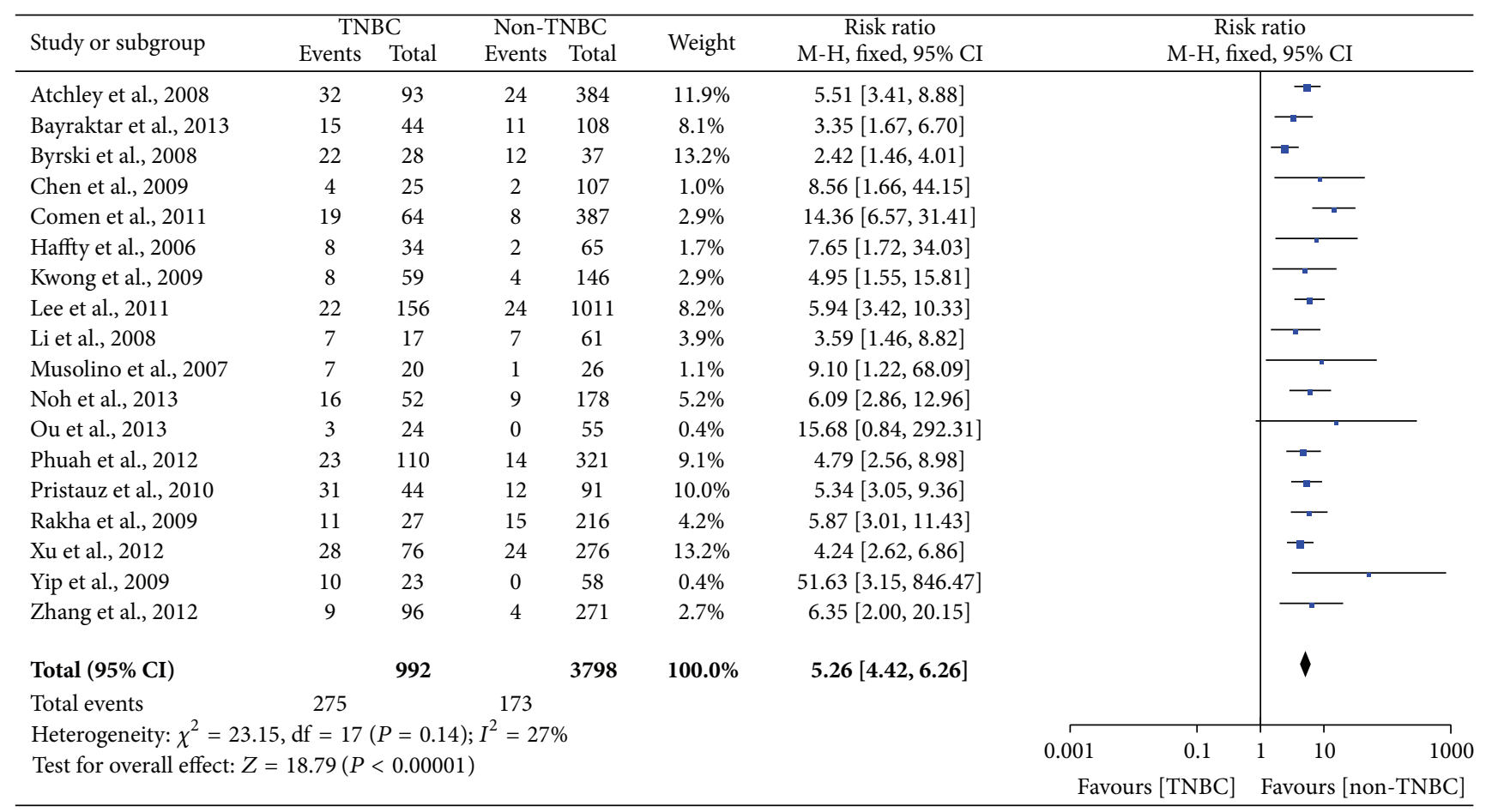

FIGURE 5: Forest plot of studies evaluating RR of BRCA1 mutation in TNBC compared with non-TNBC. The events of TNBC and the events of non-TNBC refer to the number of TNBC patients with BRCA1 mutation and the number of non-TNBC patients with BRCA1 mutation, respectively. The squares and horizontal lines correspond to the specific RR and 95\% CI for every study. The area of the squares reflects the study specific weight. The diamond stands for the pooled RR and 95\% CI.

\section{Discussion}

In this meta-analysis, the expression of $\mathrm{AR}$ and EGFR and the risk of BRCA1 mutation in TNBC are explored. We find that EGFR is overexpressed and the risk of having BRCA1 mutation is higher in TNBC than non-TNBC. Nevertheless, AR expression is downregulated in TNBC compared with nonTNBC. The distinct characteristics of AR, EGFR, and BRCA1 indicate that they can play crucial roles in targeted therapy or prognosis in TNBC.

There are some advantages in our meta-analysis. First, the most obvious superiority is that there is substantial sample size to improve the credibility for statistical analysis. Second, the conclusions are more generalizable on account of eligible literature from many different geographic distributions, such as USA, UK, Australia, and China. Finally, we extracted data information required from individual literature and composited the outcome instead of summary consequence.

However, several limitations in our meta-analysis should be discussed. First, there is extreme heterogeneity for the outcomes of AR expression $\left(P<0.00001, I^{2}=79 \%\right)$ and EGFR expression $\left(P<0.00001, I^{2}=85 \%\right)$. The detection methods of AR and EGFR are both immunohistochemistry (IHC), but the antibody source, dilution rate, cut-off value, and ethnicity are different for AR and EGFR in different studies (Tables 1 and 2). On the basis of the four factors, we proceed with subgroup analyses; only the heterogeneity about OR related to EGFR expression is markedly reduced in the subgroup connected with antibody source (Figure 4). Hence, the heterogeneity is probably attributed to the variances with regard to features of population, the subtypes of TNBC, the disease stages, the antibody source, the dilution rate, the cutoff value of AR and EGFR expression, and so forth. Second, although there is no evidence of significant publication bias for the articles about AR and EGFR in our meta-analysis, there was slight asymmetry in the funnel plot about BRCA1. Hence, cautions should be taken on the account of mild publication bias between the eligible articles about BRCA1. Besides, only the English articles were selected, which can certainly give rise to language bias. In addition, positive results are prone to be published, which may make certain bias.

As we know, TNBC is a heterogeneous disease that has high diversity associated with biology, etiology, and treatment strategies. In addition, TNBCs have a more aggressive clinical behavior in part due to poor differentiation. Furthermore, due to the lack of the conventional hormonal or anti-HER2 therapeutic targets, the standardized treatment strategies have not been formulated and the chemotherapy is the only modality of systemic therapy for these cancers. Based on the above, it is urgently needed to find new prognostic indicators and therapeutic method for TNBCs.

The meta-analysis indicates that the expression level of $\mathrm{AR}$ is lower in TNBCs than non-TNBCs (pooled OR $=0.07$, 95\% CI $=0.05-0.11$ ), but more and more studies demonstrated that about one-third of TNBCs were AR-positive [11, $23,25,27,29,30]$. This represents a potential opportunity for novel targeted therapy in positive AR expression of TNBC 


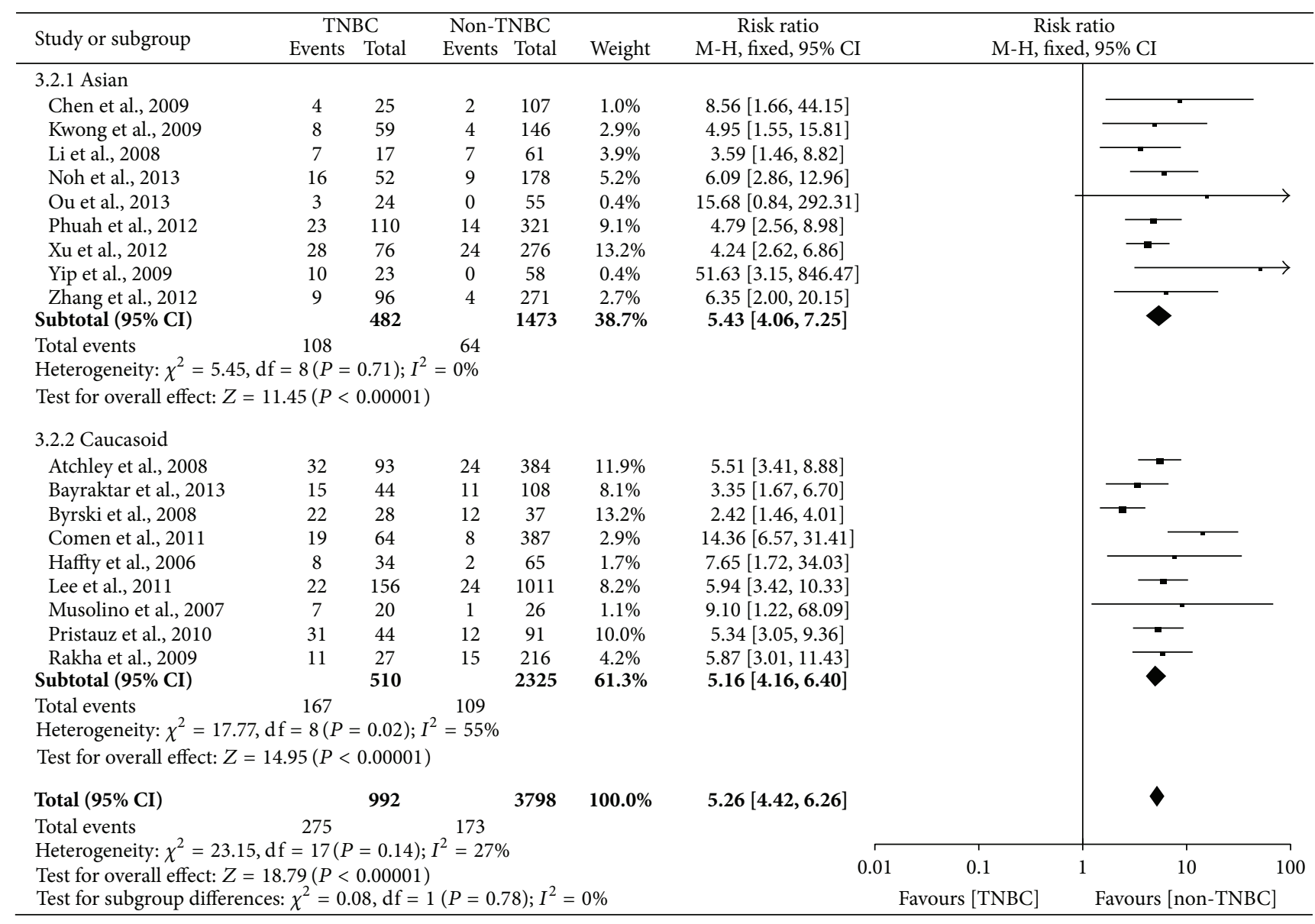

FIGURE 6: Forest plot of the subgroup related to race evaluating RR of BRCA1 mutation in TNBC compared with non-TNBC.

patients. For instance, the cell lines which had the triplenegative phenotype and AR expression had good response to bicalutamide (AR antagonist) [4]. Furthermore, more attention was paid to the association between AR expression and clinicopathological characteristics or prognostic value in ERnegative and TN breast cancers. It was consistent with the results with regard to the relationship between AR expression and clinicopathological features, and it could be summarized that positive AR immunostaining was connected with lower clinical stage, lower histological grade, and lower mitotic score $[11,23,25,29,30]$. However, the findings with respect to the association between AR expression and prognostic value were discordant. Rakha et al. [1] and Sutton et al. [12] showed that absence of AR expression was associated with the increased risk of recurrence and distant metastasis in the lymph node-positive TNBCs. Luo et al. [25] also reported that AR expression was correlated with higher 5-year disease-free survival (DFS) and overall survival (OS) of TNBC patients. In addition, the latest study [10] demonstrated that the expression level of AR was associated with better OS in the nonbasal TNBC that had no expression of basal markers. By contrast, $\mathrm{Hu}$ et al. [23] found that AR expression was associated with increased mortality among women with ER-negative and TN breast cancers. Nonetheless, there was a research showing that there was no significant association between positive AR immunostaining and DFS or OS in TNBCs [11]. Additionally, a recent study demonstrated a positive relationship between the activation of EGFR and PDGFR $\beta$ and AR expression, and inhibition of Erk1/2, EGFR, and PDGFR $\beta$ with antiandrogen bicalutamide decreased AR expression and had an additive antiproliferative effect in TNBC [7]. Taken together, these findings indicated the significance of $\mathrm{AR}$ in the initiation and progression of TNBC, and further investigation is needed to verify the function of $A R$ as a therapeutic target or prognostic marker in TNBC.

In the recent years, more studies have been carried out for the association between EGFR and various kinds of cancers. And the results were nearly consistent with our meta-analysis which showed that the EGFR expression was upregulated in TNBCs compared with non-TNBCs (pooled OR $=6.88$, 95\% CI $=3.84-12.35$ ). Based on the EGFR overexpression in TNBCs, numerous trials have been currently focusing on identifying possible therapeutic targets and prognosis for the TNBC patients with EGFR overexpression. Although the TNBC cell lines were not essentially sensitive to the EGFR inhibitor (e.g., gefitinib), gefitinib could improve response to chemotherapy. That was to say combination therapy with gefitinib and chemotherapy made a greater difference to inhibit proliferation of TNBC cells than either gefitinib or the chemotherapy alone [2]. Furthermore, Tang et al. [14] 


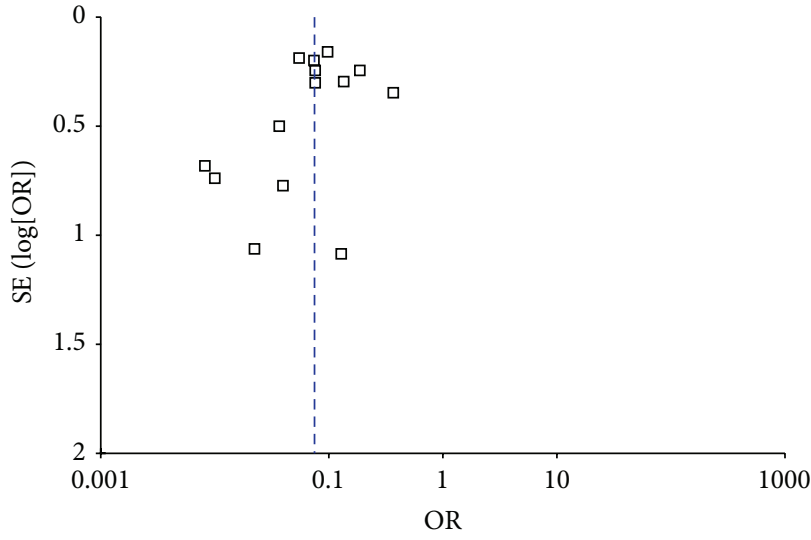

(a)

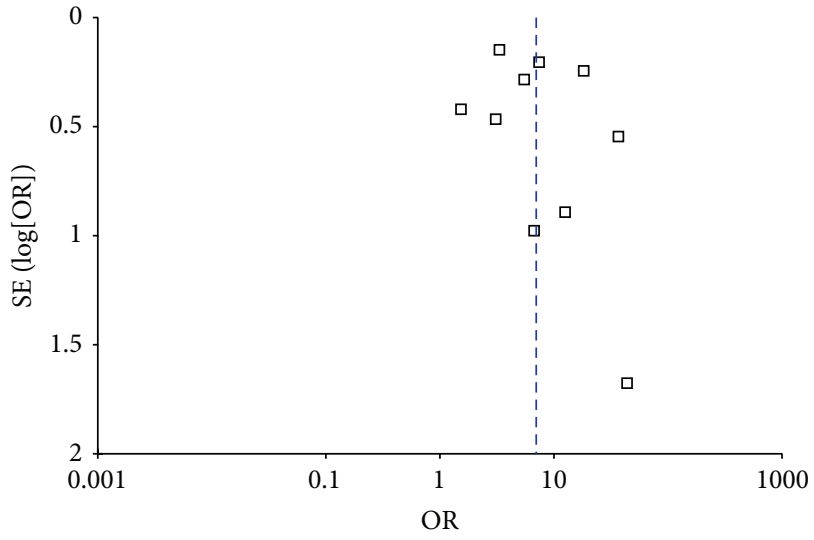

(b)

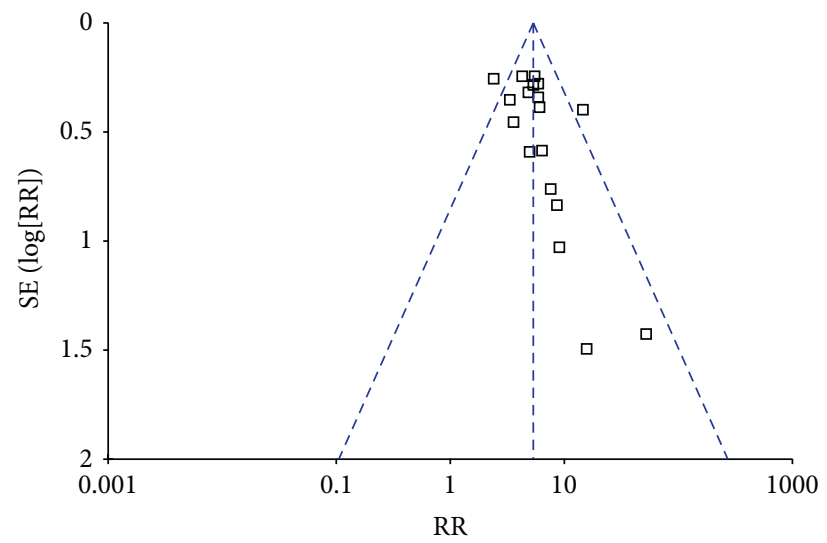

(c)

Figure 7: Funnel plots for evaluating publication bias for the eligible articles about AR (a), EGFR (b), and BRCA1 (c). As shown in the figures, the funnel plots were almost symmetric and no evidence of publication bias was observed in this analysis.

indicated that the EGFR overexpression predicted better response to neoadjuvant chemotherapy and increased pathologic complete response rates in TNBCs compared with nonTNBCs. Nonetheless, the previous study outcome demonstrated that patients with EGFR-positive TNBC had a less favorable response to neoadjuvant chemotherapy than patients with EGFR-negative TNBC [33]. In a word, the expression level of EGFR is higher in TNBCs than nonTNBCs $[1,10,14,24,33-38]$, but further studies evaluating the association between EGFR and neoadjuvant chemotherapy are required to promote molecular targeting therapy for TNBC. Furthermore, there was evidence that $94 \%$ of early-stage high-grade TNBC with a basal-like phenotype expressed MUC1, and MUC1 and EGFR interacted in nucleus of BC cell to facilitate the association of EGFR with transcriptionally active promoter regions, which provided a rationale for MUC1-based immunotherapy in TNBC patients with EGFR expression [53].

The prevalence of BRCA1 mutation in familial or earlyonset breast cancer led more and more studies to concentrate on the role of BRCA1 in TNBC $[19,40,41,43,46,49]$. The pooled RR (5.26, 95\% CI $=4.42-6.26)$ of our meta-analysis showed that the risk of BRCA1 mutation was about five times in TNBC compared with non-TNBC. Intriguingly, mounting evidence indicated that breast cancers with BRCA1 mutation were more likely to exhibit triple-negative phenotype compared with the BRCA1 noncarriers [18, 20, 40-44, 49], which showed that BRCA1 could play a unique role in the progression of TNBCs. In the subgroup regarding race, the pooled $\mathrm{RR}$ (5.43, 95\% CI $=4.06-7.25)$ for the Asian was higher than the pooled RR $(5.16,95 \% \mathrm{CI}=4.16-6.40)$ for the Caucasoid, which indicated that the prevalence of BRCA1 mutation was higher in the Asian compared with the Caucasoid. Moreover, there was no heterogeneity $\left(I^{2}=0 \%, P=0.71\right)$ in the Asian subgroup. Thus, the race was in a certain contribution to the heterogeneity and a factor causing the different prevalence of BRCA1 mutation between Caucasoid and Asian. Several studies had been conducted for the relationship related to the BRCA1-associated breast cancers and therapeutic effects. Byrski et al. [21] reported that early-onset breast cancer patients with BRCA1 mutation had poorer response to the neoadjuvant chemotherapy of the spindle poison docetaxel. Nevertheless, PARP inhibitors damaging DNA single-strand break repair could benefit patients with BRCA1 mutation [54]. However, few attentions were paid to the therapeutic effects of neoadjuvant chemotherapy in TNBC patients with 
BRCA1 mutation. Based on the above, it is necessary to further evaluate the relationship between BRCA1 mutation and TNBCs and implement the effective strategies for TNBC patients with BRCA1 mutation in the future.

In addition, some research studies have been carried out for other therapeutic target receptors (e.g., VEGFR and folate receptor) and novel inhibitors (e.g., inhibitor of $\mathrm{mTOR}$ and histone deacetylase) for the preclinical and clinical treatment of TNBC. Similar to EGFR, the vascular endothelial growth factor receptor (VEGFR) has been explored as a therapeutic target receptor in breast cancer. An open-label, randomised phase 3 trial demonstrated that bevacizumab (VEGFR inhibitor) was ineffective in adjuvant treatment in unselected TNBC patients but may have some efficacy in metastatic TNBC [55]. A recent study demonstrated that $80 \%$ of TNBC patients expressed folate receptor a (FRA) and FRA expression was significantly associated with a worse disease-free survival [56]. Additionally, the PIK3CA gene is commonly mutated in TNBC. The inhibition of the PI3K pathway and downstream mammalian target of rapamycin (mTOR) has been identified as a promising therapeutic strategy for treating TNBC, and a phase 2 trial demonstrated that everolimus (mTOR inhibitor)-carboplatin combination was efficacious in metastatic TNBC [57]. Furthermore, epigenetic alterations are known for promoter initiation and progression of cancers. Targeting such epigenetic events via histone deacetylase inhibitor (HDI) has been explored in the treatment of TNBC. A recent study showed that HDI treatment induced "BRCAness" and synergistic lethality with PARP inhibitors and cisplatin against human TNBC cells [58].

Based on the gene expression profiling, triple-negative breast cancer is a heterogeneous disease with unique molecular subtypes which have different clinicopathological features and clinical outcomes. Taken collectively, AR, EGFR, and BRCA1 play distinct roles as biomarkers in the progression of TNBCs. In view of the EGFR overexpression and the immunohistochemical feasibility of clinical practice, EGFR might be superior to AR and BRCA1 as biomarker for TNBCs. However, the unique role of AR and BRCA1 cannot be ignored for targeted treatment strategies and prognosis judgement. Furthermore, the previous study reported that $20 \%$ of the hereditary BRCA-related breast cancers had triple-negative phenotype and expressed AR [8]. To date, the relationship among the three biomarkers is not clear and further investigation should be warranted before the combination of three biomarkers is applied to the clinical management of TNBC patients.

\section{Conclusion}

Overall, by quantifying synthesis of all published studies of AR, EGFR, and BRCA1, the meta-analysis demonstrated that the expression level of EGFR and the risk of BRCA1 mutation were higher in TNBC compared with non-TNBC and AR expression was downregulated. Our study can give a valuable clue for the targeted therapy or judging prognosis of TNBC patients. More clinical research should be performed before AR, EGFR, or BRCA1 can be proved to be common biomarkers for routine clinical practices.

\section{Conflict of Interests}

The authors declare that there is no conflict of interests regarding the publication of this paper.

\section{Authors' Contribution}

Li Zhang and Cheng Fang contribute equally to this work.

\section{Acknowledgments}

The Project-sponsored by SRF for ROCS, SEM. This work was supported by grants from the National Natural Science Foundation of China (30873044 and 81272372).

\section{References}

[1] E. A. Rakha, M. E. El-Sayed, A. R. Green, A. H. S. Lee, J. F. Robertson, and I. O. Ellis, "Prognostic markers in triple-negative breast cancer," Cancer, vol. 109, no. 1, pp. 25-32, 2007.

[2] B. Corkery, J. Crown, M. Clynes, and N. O’Donovan, "Epidermal growth factor receptor as a potential therapeutic target in triple-negative breast cancer," Annals of Oncology, vol. 20, no. 5, pp. 862-867, 2009.

[3] B. G. Haffty, Q. Yang, M. Reiss et al., "Locoregional relapse and distant metastasis in conservatively managed triple negative early-stage breast cancer," Journal of Clinical Oncology, vol. 24, no. 36, pp. 5652-5657, 2006.

[4] B. D. Lehmann, J. A. Bauer, X. Chen et al., "Identification of human triple-negative breast cancer subtypes and preclinical models for selection of targeted therapies," The Journal of Clinical Investigation, vol. 121, no. 7, pp. 2750-2767, 2011.

[5] L. Steward, L. Conant, F. Gao, and J. A. Margenthaler, "Predictive factors and patterns of recurrence in patients with triple negative breast cancer," Annals of Surgical Oncology, vol. 21, no. 7, pp. 2165-2171, 2014.

[6] J. J. Park, R. A. Irvine, G. Buchanan et al., "Breast cancer susceptibility gene 1 (BRCA1) is a coactivator of the androgen receptor," Cancer Research, vol. 60, no. 21, pp. 5946-5949, 2000.

[7] M. D. Cuenca-López, J. C. Montero, J. C. Morales, A. Prat, A. Pandiella, and A. Ocana, "Phospho-kinase profile of triple negative breast cancer and androgen receptor signaling," $B M C$ Cancer, vol. 14, no. 1, pp. 302-311, 2014.

[8] G. Pristauz, E. Petru, E. Stacher et al., "Androgen receptor expression in breast cancer patients tested for BRCA1 and BRCA2 mutations," Histopathology, vol. 57, no. 6, pp. 877-884, 2010.

[9] A. Gucalp and T. A. Traina, "Triple-negative breast cancer: role of the androgen receptor," Cancer Journal, vol. 16, no. 1, pp. 6265, 2010.

[10] P. Gasparini, M. Fassan, L. Gascione et al., "Androgen receptor status is a prognostic marker in non-basal triple negative breast cancers and determines novel therapeutic options," PLOS ONE, vol. 9, no. 2, Article ID e88525, 2014.

[11] I. Mrklić, Z. Pogorelić, V. Ćapkun, and S. Tomić, "Expression of androgen receptors in triple negative breast carcinomas," Acta Histochemica, vol. 115, no. 4, pp. 344-348, 2013.

[12] L. M. Sutton, D. Cao, V. Sarode et al., "Decreased androgen receptor expression is associated with distant metastases in patients with androgen receptor-expressing triple-negative breast carcinoma," The American Journal of Clinical Pathology, vol. 138, no. 4, pp. 511-516, 2012. 
[13] D. Tang, S. Xu, Q. Zhang, and W. Zhao, "The expression and clinical significance of the androgen receptor and E-cadherin in triple-negative breast cancer," Medical Oncology, vol. 29, no. 2, pp. 526-533, 2012.

[14] Y. Tang, L. Zhu, Y. Li et al., "Overexpression of epithelial growth factor receptor (EGFR) predicts better response to neo-adjuvant chemotherapy in patients with triple-negative breast cancer," Journal of Translational Medicine, vol. 10, supplement 1, article S4, 2012.

[15] J.-S. Guillamo, S. de Boüard, S. Valable et al., "Molecular mechanisms underlying effects of epidermal growth factor receptor inhibition on invasion, proliferation, and angiogenesis in experimental glioma," Clinical Cancer Research, vol. 15, no. 11, pp. 3697-3704, 2009.

[16] A. R. Venkitaraman, "Cancer susceptibility and the functions of BRCA1 and BRCA2," Cell, vol. 108, no. 2, pp. 171-182, 2002.

[17] J. S. Reis-Filho and A. N. Tutt, "Triple negative tumours: a critical review," Histopathology, vol. 52, no. 1, pp. 108-118, 2008.

[18] D. P. Atchley, C. T. Albarracin, A. Lopez et al., "Clinical and pathologic characteristics of patients with BRCA-positive and BRCA-negative breast cancer," Journal of Clinical Oncology, vol. 26, no. 26, pp. 4282-4288, 2008.

[19] A. Musolino, M. A. Bella, B. Bortesi et al., "BRCA mutations, molecular markers, and clinical variables in early-onset breast cancer: a population-based study," Breast, vol. 16, no. 3, pp. $280-$ 292, 2007.

[20] W.-F. Li, Z. Hu, N.-Y. Rao et al., "The prevalence of BRCA1 and $B R C A 2$ germline mutations in high-risk breast cancer patients of Chinese Han nationality: two recurrent mutations were identified," Breast Cancer Research and Treatment, vol. 110, no. 1, pp. 99-109, 2008.

[21] T. Byrski, J. Gronwald, T. Huzarski et al., "Response to neoadjuvant chemotherapy in women with BRCAl-positive breast cancers," Breast Cancer Research and Treatment, vol. 108, no. 2, pp. 289-296, 2008.

[22] B. J. Chae, A. Lee, J. S. Bae, B. J. Song, and S. S. Jung, "Expression of nuclear receptor DAX-1 and androgen receptor in human breast cancer," Journal of Surgical Oncology, vol. 103, no. 8, pp. 768-772, 2011.

[23] R. Hu, S. Dawood, M. D. Holmes et al., "Androgen receptor expression and breast cancer survival in postmenopausal women," Clinical Cancer Research, vol. 17, no. 7, pp. 1867-1874, 2011.

[24] J. S. Koo and W. Jung, "Clinicopathlogic and immunohistochemical characteristics of triple negative invasive lobular carcinoma," Yonsei Medical Journal, vol. 52, no. 1, pp. 89-97, 2011.

[25] X. Luo, Y.-X. Shi, Z.-M. Li, and W.-Q. Jiang, "Expression and clinical significance of androgen receptor in triple negative breast cancer," Chinese Journal of Cancer, vol. 29, no. 6, pp. 585590, 2010.

[26] S. Loibl, B. M. Müller, G. von Minckwitz et al., "Androgen receptor expression in primary breast cancer and its predictive and prognostic value in patients treated with neoadjuvant chemotherapy," Breast Cancer Research and Treatment, vol. 130, no. 2, pp. 477-487, 2011.

[27] D. Micello, A. Marando, N. Sahnane, C. Riva, C. Capella, and F. Sessa, "Androgen receptor is frequently expressed in HER2positive, ER/PR-negative breast cancers," Virchows Archiv, vol. 457, no. 4, pp. 467-476, 2010.

[28] L. A. Niemeier, D. J. Dabbs, S. Beriwal, J. M. Striebel, and R. Bhargava, "Androgen receptor in breast cancer: expression in estrogen receptor-positive tumors and in estrogen receptornegative tumors with apocrine differentiation," Modern Pathology, vol. 23, no. 2, pp. 205-212, 2010.

[29] Y. Ogawa, E. Hai, K. Matsumoto et al., "Androgen receptor expression in breast cancer: relationship with clinicopathological factors and biomarkers," International Journal of Clinical Oncology, vol. 13, no. 5, pp. 431-435, 2008.

[30] S. Park, J. Koo, H. S. Park et al., "Expression of androgen receptors in primary breast cancer," Annals of Oncology, vol. 21, no. 3, pp. 488-492, 2009.

[31] S. Park, J. S. Koo, M. S. Kim et al., "Androgen receptor expression is significantly associated with better outcomes in estrogen receptor-positive breast cancers," Annals of Oncology, vol. 22, no. 8, pp. 1755-1762, 2011.

[32] K. M. Peters, S. L. Edwards, S. S. Nair et al., "Androgen receptor expression predicts breast cancer survival: the role of genetic and epigenetic events," BMC Cancer, vol. 12, pp. 132-141, 2012.

[33] H. Nogi, T. Kobayashi, M. Suzuki et al., "EGFR as paradoxical predictor of chemosensitivity and outcome among triplenegative breast cancer," Oncology Reports, vol. 21, no. 2, pp. 413417, 2009.

[34] T. Nozoe, E. Mori, T. Iguchi et al., "Immunohistochemical expression of epidermal growth factor receptor in breast cancer," Breast Cancer, vol. 18, no. 1, pp. 37-41, 2011.

[35] S. K. K. Pillai, A. Tay, S. Nair, and C.-O. Leong, "Triple-negative breast cancer is associated with EGFR, CK5/6 and c-KIT expression in Malaysian women," BMC Clinical Pathology, vol. 12, article 18, 2012.

[36] L. Rydén, K. Jirstrom, M. Haglund, O. Stal, and M. Fernö, "Epidermal growth factor receptor and vascular endothelial growth factor receptor 2 are specific biomarkers in triple-negative breast cancer. Results from a controlled randomized trial with long-term follow-up," Breast Cancer Research and Treatment, vol. 120, no. 2, pp. 491-498, 2010.

[37] D. S. P. Tan, C. Marchió, R. L. Jones et al., “Triple negative breast cancer: molecular profiling and prognostic impact in adjuvant anthracycline-treated patients," Breast Cancer Research and Treatment, vol. 111, no. 1, pp. 27-44, 2008.

[38] O. Tawfik, K. Davis, B. F. Kimler et al., "Clinicopathological characteristics of triple-negative invasive mammary carcinomas in African-American versus Caucasian women," Annals of Clinical and Laboratory Science, vol. 40, no. 4, pp. 315-323, 2010.

[39] S. Bayraktar, A. M. Gutierrez-Barrera, H. Lin et al., "Outcome of metastatic breast cancer in selected women with or without deleterious BRCA mutations," Clinical and Experimental Metastasis, vol. 30, no. 5, pp. 631-642, 2013.

[40] W. Chen, K. Pan, T. Ouyang et al., "BRCA1 germline mutations and tumor characteristics in Chinese women with familial or early-onset breast cancer," Breast Cancer Research and Treatment, vol. 117, no. 1, pp. 55-60, 2009.

[41] E. Comen, M. Davids, T. Kirchhoff, C. Hudis, K. Offit, and M. Robson, "Relative contributions of BRCA1 and BRCA2 mutations to "triple-negative" breast cancer in Ashkenazi Women," Breast Cancer Research and Treatment, vol. 129, no. 1, pp. 185190, 2011.

[42] A. Kwong, L. P. Wong, H. N. Wong et al., "Clinical and pathological characteristics of Chinese patients with BRCA related breast cancer," The HUGO Journal, vol. 3, no. 1, pp. 63-76, 2009.

[43] E. Lee, R. McKean-Cowdin, H. Ma et al., "Characteristics of triple-negative breast cancer in patients with a BRCA1 mutation: results from a population-based study of young women," Journal of Clinical Oncology, vol. 29, no. 33, pp. 4373-4380, 2011. 
[44] J. M. Noh, B.-K. Han, D. H. Choi et al., "Association between BRCA mutation status, pathological findings, and magnetic resonance imaging features in patients with breast cancer at risk for the mutation," Journal of Breast Cancer, vol. 16, no. 3, pp. 308-314, 2013.

[45] J. Ou, T. Wu, R. Sijmons, D. Ni, W. Xu, and H. Upur, "Prevalence of BRCA1 and BRCA2 germline mutations in breast cancer women of multiple ethnic region in Northwest China," Journal of Breast Cancer, vol. 16, no. 1, pp. 50-54, 2013.

[46] S.-Y. Phuah, L.-M. Looi, N. Hassan et al., "Triple-negative breast cancer and PTEN (phosphatase and tensin homologue)loss are predictors of BRCA1 germline mutations in women with earlyonset and familial breast cancer, but not in women with isolated late-onset breast cancer," Breast Cancer Research, vol. 14, no. 6, article R142, 2012.

[47] J. Xu, B. Wang, Y. Zhang, R. Li, Y. Wang, and S. Zhang, "Clinical implications for BRCA gene mutation in breast cancer," Molecular Biology Reports, vol. 39, no. 3, pp. 3097-3102, 2012.

[48] C.-H. Yip, N. A. Taib, W. Y. Choo, S. Rampal, M. K. Thong, and S. H. Teo, "Clinical and pathologic differences between BRCA1-, $B R C A 2-$, and non-BRCA-associated breast cancers in a multiracial developing country," World Journal of Surgery, vol. 33, no. 10, pp. 2077-2081, 2009.

[49] J. Zhang, R. Pei, Z. Pang et al., "Prevalence and characterization of BRCA1 and BRCA2 germline mutations in Chinese women with familial breast cancer," Breast Cancer Research and Treatment, vol. 132, no. 2, pp. 421-428, 2012.

[50] E. A. Rakha, S. E. Elsheikh, M. A. Aleskandarany et al., "Triple-negative breast cancer: distinguishing between basal and nonbasal subtypes," Clinical Cancer Research, vol. 15, no. 7, pp. 2302-2310, 2009.

[51] J. P. T. Higgins and S. G. Thompson, "Quantifying heterogeneity in a meta-analysis," Statistics in Medicine, vol. 21, no. 11, pp. 15391558, 2002.

[52] J. P. T. Higgins, S. G. Thompson, J. J. Deeks, and D. G. Altman, "Measuring inconsistency in meta-analyses," British Medical Journal, vol. 327, no. 7414, pp. 557-560, 2003.

[53] A. Siroy, F. W. Abdul-Karim, J. Miedler et al., "MUC1 is expressed at high frequency in early-stage basal-like triple negative breast cancer," Human Pathology, vol. 44, no. 10, pp. 2159-2166, 2013.

[54] O. Gluz, C. Liedtke, N. Gottschalk, L. Pusztai, U. Nitz, and N. Harbeck, "Triple-negative breast cancer: current status and future directions," Annals of Oncology, vol. 20, no. 12, pp. 19131927, 2009.

[55] D. Cameron, J. Brown, R. Dent et al., "Adjuvant bevacizumabcontaining therapy in triple-negative breast cancer (BEATRICE): primary results of a randomised, phase 3 trial," The Lancet Oncology, vol. 14, no. 10, pp. 933-942, 2013.

[56] Z. Zhang, J. Wang, D. E. Tacha et al., "Folate receptor $\alpha$ associate with triple-negative breast cancer and poor prognosis," Archives of Pathology \& Laboratory Medicine, vol. 138, no. 7, pp. 890-895, 2014.

[57] J. C. Singh, Y. Novik, S. Stein et al., "Phase 2 trial of everolimus and carboplatin combination in patients with triple negative metastatic breast cancer," Breast Cancer Research, vol. 16, no. 2, article R32, 2014.

[58] K. Ha, W. Fiskus, D. S. Choi et al., "Histone deacetylase inhibitor treatment induces "BRCAness" and synergistic lethality with PARP inhibitor and cisplatin against human triple negative breast cancer cells," Oncotarget, vol. 5, no. 14, pp. 5637-5650, 2014. 


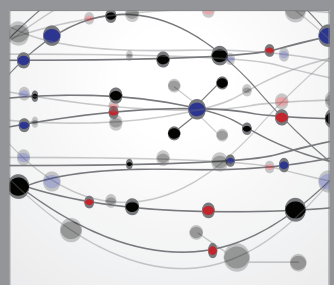

The Scientific World Journal
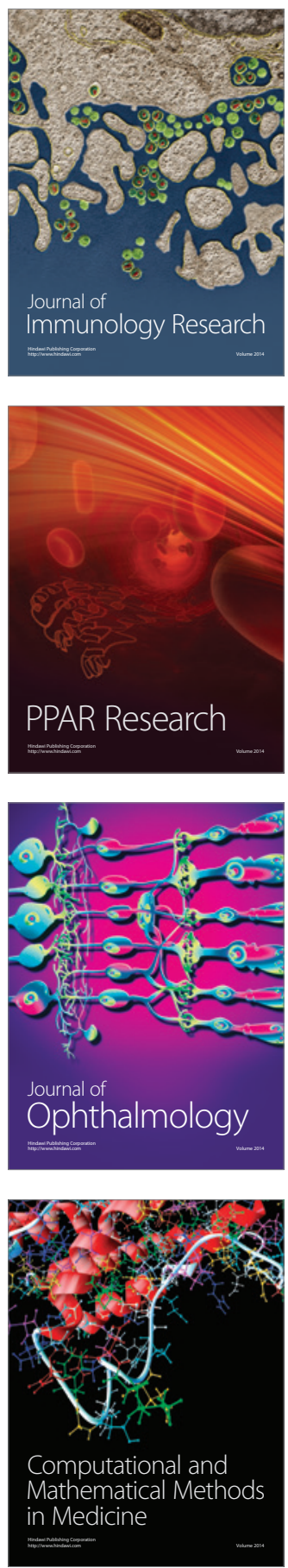

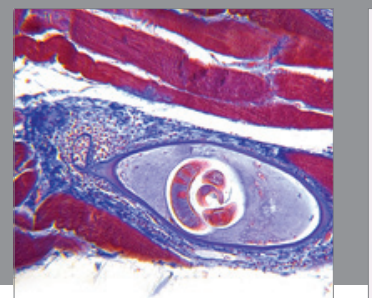

Gastroenterology

Research and Practice
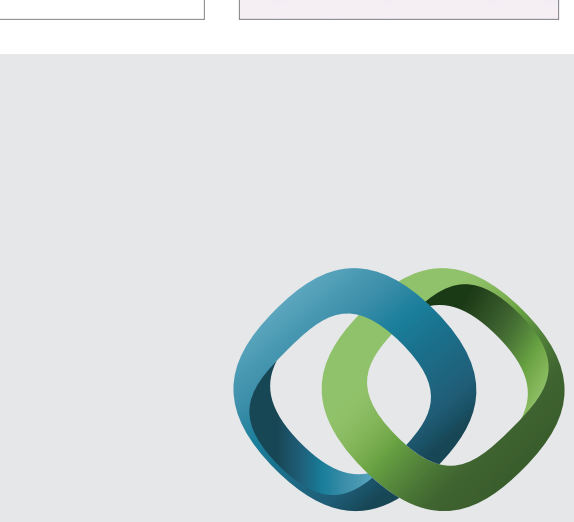

\section{Hindawi}

Submit your manuscripts at

http://www.hindawi.com
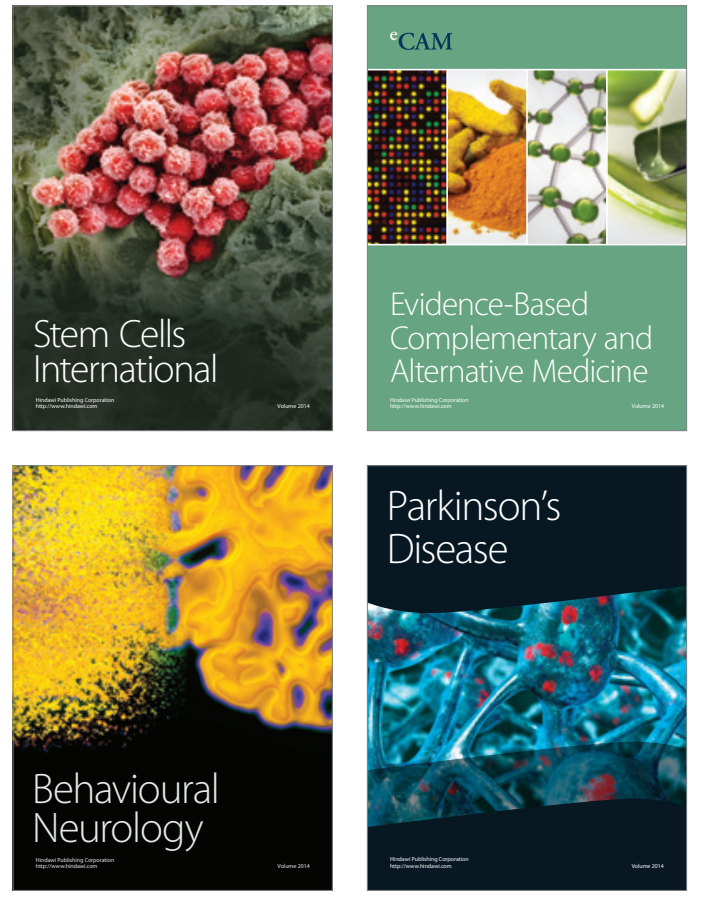
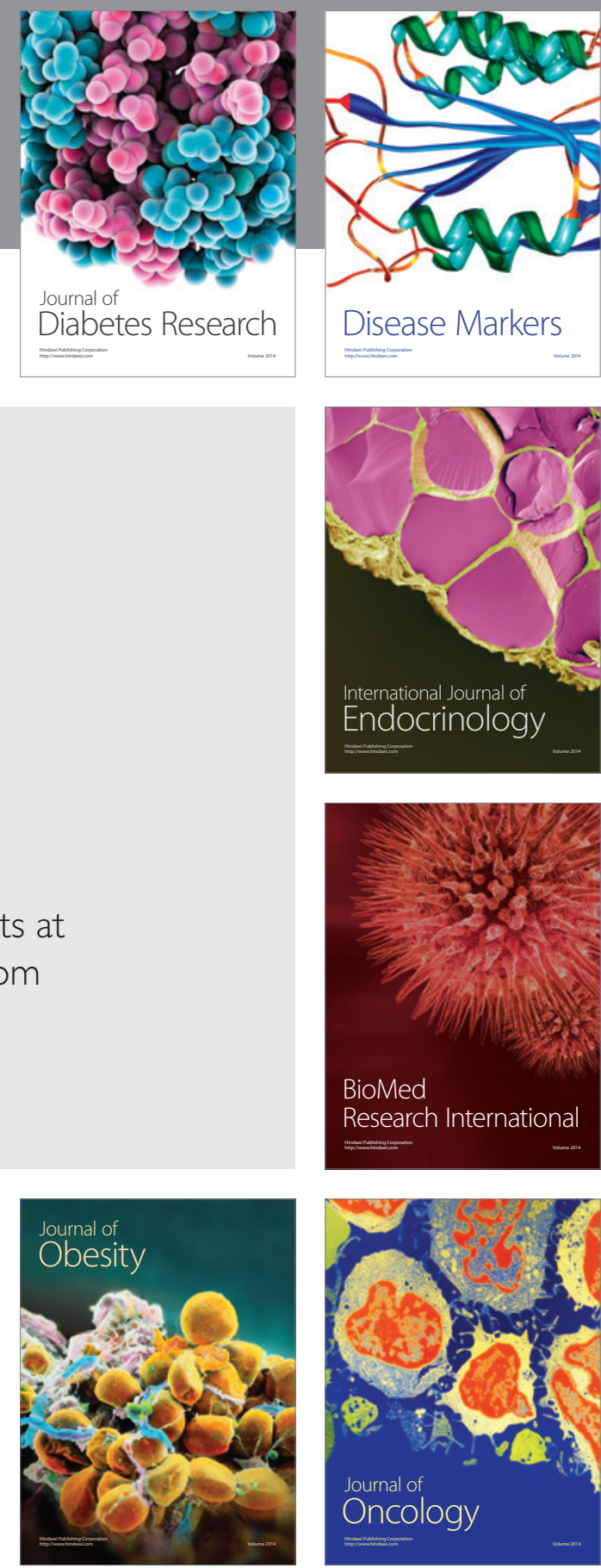

Disease Markers
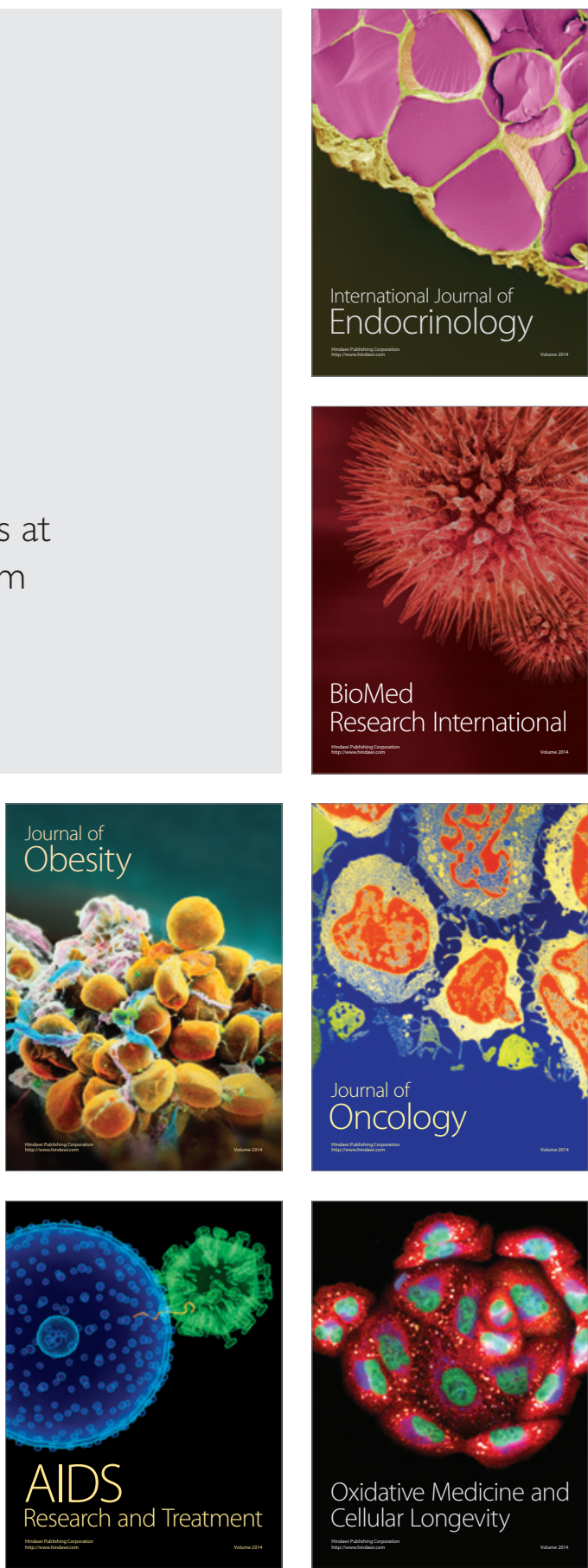